\title{
Systematic design of loss-engineered slow-light waveguides
}

\author{
Wang, Fengwen; Jensen, Jakob Søndergaard; Mørk, Jesper; Sigmund, Ole
}

Published in:

Optical Society of America. Journal A: Optics, Image Science, and Vision

Publication date:

2012

Document Version

Publisher's PDF, also known as Version of record

Link back to DTU Orbit

Citation (APA):

Wang, F., Jensen, J. S., Mørk, J., \& Sigmund, O. (2012). Systematic design of loss-engineered slow-light waveguides. Optical Society of America. Journal A: Optics, Image Science, and Vision, 29(12), 2657-2666.

\section{General rights}

Copyright and moral rights for the publications made accessible in the public portal are retained by the authors and/or other copyright owners and it is a condition of accessing publications that users recognise and abide by the legal requirements associated with these rights.

- Users may download and print one copy of any publication from the public portal for the purpose of private study or research.

- You may not further distribute the material or use it for any profit-making activity or commercial gain

- You may freely distribute the URL identifying the publication in the public portal

If you believe that this document breaches copyright please contact us providing details, and we will remove access to the work immediately and investigate your claim. 


\title{
Systematic design of loss-engineered slow-light waveguides
}

\author{
Fengwen Wang, ${ }^{1, *}$ Jakob Søndergaard Jensen, ${ }^{1}$ Jesper Mørk, ${ }^{2}$ and Ole Sigmund ${ }^{1}$ \\ ${ }^{1}$ Department of Mechanical Engineering, Technical University of Denmark, \\ Nils Koppels Allé, Building 404, 2800 Kgs. Lyngby, Denmark \\ ${ }^{2}$ Department of Photonics Engineering, Technical University of Denmark, Ørsteds Plads, \\ Building 345, 2800 Kgs. Lyngby, Denmark \\ *Corresponding author: fwan@mek.dtu.dk
}

Received July 11, 2012; accepted September 21, 2012;

posted October 24, 2012 (Doc. ID 172342); published November 28, 2012

\begin{abstract}
This paper employs topology optimization to systematically design free-topology loss-engineered slow-light waveguides with enlarged group index bandwidth product (GBP). The propagation losses of guided modes are evaluated by the imaginary part of eigenvalues in complex band structure calculations, where the scattering losses due to manufacturing imperfections are represented by an edge-related effective dissipation. The loss engineering of slow-light waveguides is realized by minimizing the propagation losses of design modes. Numerical examples illustrate that the propagation losses of free-topology dispersion-engineered waveguides can be significantly suppressed by loss engineering. Comparisons between fixed-and free-topology loss-engineered waveguides demonstrate that the GBP can be enhanced significantly by the free-topology loss-engineered waveguides with a small increase of the propagation losses. (c) 2012 Optical Society of America OCIS codes: $\quad 000.4430,130.5296,230.7390$.
\end{abstract}

\section{INTRODUCTION}

In the past decade, photonic crystal waveguides (PhCWs), i.e., photonic crystals (PhCs) with line defects, have attained extensive interest due to their capability in manipulating light propagation. Approaching the band edge, the group refractive index increases and slow-light propagation effects prevail, with a number of possible applications, e.g., for tunable delays [1,ㄹ], enhanced light-matter interaction, optical switching [3] , nonlinear optics $[\underline{4}, \underline{5}]$, and mode-locked lasers []ㅡ. It has been reported that both material loss and material gain in the PhCWs restrict the attainable group index and degrade slowlight effects $[7,8]$. Moreover, the benefits of slow light are typically compromised by significant group velocity dispersion (GVD) [9] and concomitant propagation losses $[\underline{10}, 11]$.

Several approaches have been investigated numerically and experimentally to engineer the dispersion of slow-light modes and optimize the group index bandwidth products (GBPs), namely, dispersion compensation in the coupled PhCWs $[12,13]$ and dispersion curve engineering [14-16]. Dispersion compensation has been realized in $\mathrm{PhC}$ coupled waveguides through adiabatic geometry change, and a GBP of 0.38 has been experimentally achieved [17]. Dispersion curve engineering was initially performed using trial-and-error methods guided by physical intuition [14-16], i.e., changing radii and locations of air holes and, recently, a material mask overlay strategy (MMOS) was used by some of the authors to optimize fixed-topology waveguides (circular-hole-based waveguides) using a gradient-based algorithm [18]. Through distributing dielectric material in the periodic supercell, a GBP of 0.47 for group index above 200 can be achieved by free-topology waveguides using topology optimization $[19,20]$, and, furthermore, the robustness of waveguides has been enhanced with respect to manufacturing imperfections through a robust optimization approach [20,21].

The propagation losses in PhCWs are critical for the application of slow-light waveguides and have received growing interest. Even though slow-light propagation in PhCWs is intrinsically lossless below the light line, fabricated waveguides display significant extrinsic losses due to manufacturing imperfections. The manufacturing imperfections, i.e., variations of vertical wall surface roughness, cause significant out-of-plane radiation $[\underline{22}, \underline{23}]$ and backscattering $[\underline{24}, \underline{25}]$, and, hence, lead to large propagation losses, especially for slow light. The out-of-plane radiation has been reported to follow a $n_{g}$ scaling [26]. With a theoretical model based on the Green function tensor (GFT), the backscattering was found to follow a $n_{g}^{2}$ scaling [10,24,27], which has been experimentally confirmed [11]. More recently, Petrov et al. numerically demonstrated that the propagation losses vary in different designs due to the different field intensity on the interfaces between air and dielectric material [28]. This observation has been experimentally verified [25]. These very first studies have opened up new prospects in the loss engineering of slow-light waveguides.

In this paper, we explore the use of a systematic (inverse) method for designing waveguides with minimum propagation loss due to fabrication-related disorder effects. While the disorder-induced losses originate from random variations between the unit cells of the waveguide, we here assume that those losses will be minimized if the optical intensity overlap with the hole edges is minimized [28]. We thus, phenomenologically, introduce an edge loss to represent the scattering losses, modeled by an imaginary part of the permittivity $[22,23,29]$. The propagation losses in the waveguides are directly evaluated by the solution of the complex band structure of the waveguide supercell. We use the MMOS and the density approach to 
parameterize the periodic supercell and design both fixedand free-topology waveguides. We demonstrate that the propagation losses in the free-topology waveguides can be significantly reduced while retaining the enlarged GBP. Compared to the fixed-topology loss-engineered waveguides, the enhancement of GBP in the free-topology loss-engineered waveguides induces an acceptable increase of the propagation losses.

The paper is organized as follows. In Section 2, we introduce the governing equations for the complex band structure calculation and corresponding finite element formulation. We then discuss the optimization formulations for waveguide engineering including dispersion engineering and loss engineering based on two different parameterizations of the periodic supercell. In Section 3 , we investigate the propagation losses of different waveguides and design fixed- and free-topology loss-engineered waveguides for different group indices. We summarize our studies in Section $\underline{4}$.

\section{OPTIMIZATION PROBLEMS OF SLOW-LIGHT WAVEGUIDES}

\section{A. Complex Band Structure Calculation}

Assuming TE-polarized light in a two-dimensional (2D) PhCW, the time harmonic modal profile with time dependence as $\exp (i \omega t)$ obeys the scalar Helmholtz equation:

$$
\nabla \cdot\left(\varepsilon_{r}^{-1} \nabla h\right)+(\omega / c)^{2} \mu_{r} h=0 .
$$

Here $h$ is the magnetic field in the out-of-plane direction, $\varepsilon_{r}$ is the relative permittivity, $\omega$ is the angular wave frequency, $c$ is the speed of light in vacuum, and $\mu_{r}=1$ is the relative permeability. In order to tackle the losses encountered by slow light in waveguides, we employ a phenomenological approach [22] and represent the losses using an effective dissipation by including an imaginary part of the permittivity [7], i.e., $\varepsilon_{r}=\varepsilon_{r}^{\prime}+i \varepsilon_{r}^{\prime \prime}$.

In a periodic medium, $\varepsilon_{r}(\mathbf{r}+\mathbf{a})=\varepsilon_{r}(\mathbf{r})$, with $\mathbf{r}=\left[x_{1}, x_{2}\right]$ being the position vector and $\mathbf{a}$ as the periodic vector, the solution of the above equation can be expressed in terms of the wave vector $\mathbf{k}$ following Bloch's theorem, $h(\mathbf{r})=$ $u(\mathbf{r}) \exp (i \mathbf{k} \cdot \mathbf{r})$. This leads to

$$
(\nabla+i \mathbf{k}) \cdot \varepsilon_{r}^{-1}(\nabla+i \mathbf{k}) u+(\omega / c)^{2} \mu_{r} u=0,
$$

with the periodic boundary conditions $u(\mathbf{k}, \mathbf{r}+\mathbf{a})=u(\mathbf{k}, \mathbf{r})$.

Using the finite element method (FEM) discretization procedure, Eq. (2) results in a quadratic eigenvalue equation in the longitudinal wavenumber $k$,

$$
\left(\mathbf{K}_{0}-\omega^{2} \mathbf{M}\right) \mathbf{u}+\mathbf{K}_{1} k \mathbf{u}+\mathbf{K}_{2} k^{2} \mathbf{u}=0
$$

The elementwise quantities in Eq. (3) are calculated by

$$
\begin{aligned}
\mathbf{K}_{0} & =\sum_{e} \varepsilon_{r}^{-1} \int_{e}\left(\frac{\partial \mathbf{N}^{T}}{\partial x_{1}} \frac{\partial \mathbf{N}}{\partial x_{1}}+\frac{\partial \mathbf{N}^{T}}{\partial x_{2}} \frac{\partial \mathbf{N}}{\partial x_{2}}\right) \mathrm{d} V, \\
\mathbf{M} & =\sum_{e} \mu_{r} / c^{2} \int_{e}\left(\mathbf{N}^{T} \mathbf{N}\right) \mathrm{d} V \\
\mathbf{K}_{1} & =\sum_{e} \varepsilon_{r}^{-1} i \int_{e}\left(\mathbf{N}^{T} \frac{\partial \mathbf{N}}{\partial x_{2}}-\frac{\partial \mathbf{N}^{T}}{\partial x_{2}} \mathbf{N}\right) \mathrm{d} V \\
\mathbf{K}_{2} & =\sum_{e} \varepsilon_{r}^{-1} \int_{e}\left(\mathbf{N}^{T} \mathbf{N}\right) \mathrm{d} V
\end{aligned}
$$

with $\mathbf{N}$ being the standard finite element shape functions and the waveguide being directed along $x_{2}$.

Equation (3) can be solved for the eigenfrequencies $\omega$ for a given wavenumber $k$, called the $\omega(k)$ formulation, which corresponds to a linear eigenvalue problem for $\omega^{2}$. Alternatively, this equation can also be solved for wavenumbers $k$ for a given frequency $\omega$, which results in a quadratic eigenvalue problem for $k$, called the $k(\omega)$ formulation. The detailed comparison between these two methods can be found in [30]. Here, we focus on the $k(\omega)$ formulation because of the straightforward way in which it can handle material dispersion and attenuation.

In the $k(\omega)$ formulation, Eq. (3) is solved by reformulating the equation into a linear eigenvalue problem in terms of $k$,

$$
\left(\mathbf{K}_{l}-k \mathbf{M}_{l}\right) \mathbf{U}=0,
$$

with

$$
\begin{aligned}
& \mathbf{K}_{l}=\left[\begin{array}{cc}
-\mathbf{K}_{1} & -\left(\mathbf{K}_{0}-\omega^{2} \mathbf{M}\right) \\
\mathbf{I} & 0
\end{array}\right], \\
& \mathbf{M}_{l}=\left[\begin{array}{cc}
\mathbf{K}_{2} & 0 \\
0 & \mathbf{I}
\end{array}\right], \quad \mathbf{U}=\left[\begin{array}{c}
k \mathbf{u} \\
\mathbf{u}
\end{array}\right] .
\end{aligned}
$$

The eigenvalues $k$ found from Eq. (5) are complex-valued with the real part of the eigenvalue, $\mathfrak{R}(k)$, being the propagation constant and the imaginary part of the eigenvalue, $\mathfrak{I}(k)$, representing the spatial attenuation of the wave at the given frequency.

Figure 1(b) depicts the band structure of a PhCW with triangular patterned air holes of $r=0.3 a$ in silicon (Si) [seen in Fig. 1(a)]. The left panel shows in solid curves the band structure calculated using the $\omega(k)$ formulation, while the symbols show the calculated band structure based on the $k(\omega)$ formulation. The right panel shows the propagation loss of eigenmodes in the $k(\omega)$ formulation. Different from the $\omega(k)$ formulation, many other modes are detected with the $k(\omega)$ formulation. According to the characteristics of the eigenmodes, they can be classified into two catagories: guided modes, represented by asterisks, and evanescent modes, in circles, connecting the inflection points $\left(v_{g}=0\right)$ in different bands. The evanescent and guided modes can be easily identified

(a)

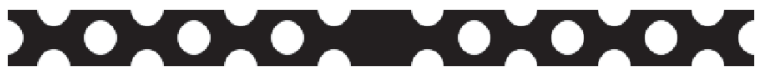

(b)

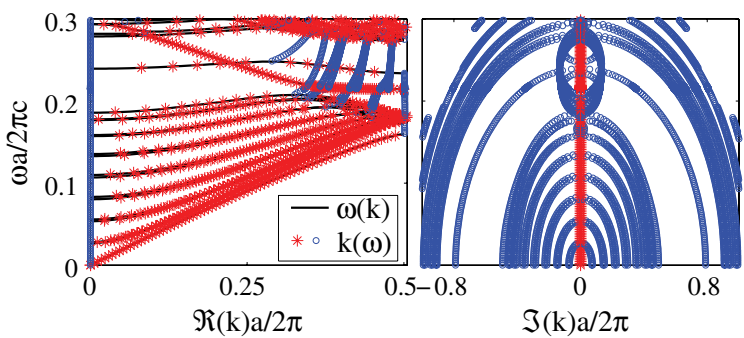

Fig. 1. (Color online) Band structure calculations. (a) Supercell. (b) Complex band structure. The solid curves represent the band structure calculated using the $\omega(k)$ formulation, and the symbols represent the band structure calculated using the $k(\omega)$ formulation, where the asterisks denote the guided modes and the circles denote the evanescent modes. 
according to their propagation loss since the evanescent modes exhibit much larger propagation loss than the guided ones.

\section{B. Influence of Material Loss/Gain on the Slow-Light Waveguides}

The group index of the gap-guided modes is defined as

$$
n_{g}=\frac{c}{v_{g}}=\mathfrak{R}\left(\frac{\partial k}{\partial \omega}\right)
$$

where $n_{g}$ is the group index of the guided modes, $v_{g}$ is the group velocity of the guided modes, and $\mathfrak{R}(\cdot)$ indicates the real part of the value.

Numerically, the derivative of eigenvalue $k$ with respect to $\omega$ can be calculated by

$$
\frac{\partial k}{\partial \omega}=\left(\mathbf{U}_{0}^{\prime}\left(\frac{\partial \mathbf{K}_{l}}{\partial \omega}-k \frac{\partial \mathbf{M}_{l}}{\partial \omega}\right) \mathbf{U}\right)
$$

where the prime denotes the conjugate transpose and $\mathbf{U}_{0}^{\prime}$ is the left eigenvector of the eigenvalue problem in Eq. (5), obtained by solving

$$
\left(\mathbf{K}_{l}^{\prime}-k^{\prime} \mathbf{M}_{l}^{\prime}\right) \mathbf{U}_{0}=0
$$

The left eigenvector $\mathbf{U}_{0}^{\prime}$ and the right eigenvector $\mathbf{U}$ are normalized with respect to $\mathbf{M}_{l}$ :

$$
\mathbf{U}_{0}^{\prime} \mathbf{M}_{l} \mathbf{U}=1
$$

Figure 2(a) shows the gap-guided bands and corresponding group indices in the presence of different amounts of material loss $\left(\varepsilon_{\mathrm{Si}}=\left(n+\mathrm{in}^{\prime \prime}\right)^{2}\right)$. The material loss causes the guided band to deviate from a parabolic shape, as in the lossless case when approaching the band edge, and, hence, restricts the achievable group index [7]. Furthermore, a redshift is observed in the gap-guided band due to material loss.

It should be noted that material gain $\left(n^{\prime \prime}<0\right)$ [shown in Fig. 2(b)] exhibits the same influence on the achievable group index of waveguides as the corresponding loss; i.e., only the magnitude of $n^{\prime \prime}$ matters [8]. This conclusion can be deduced simply from Eq. (3). If we consider the same amount of material gain as material loss, all the matrices in Eq. (3) are conjugate transposes of the ones for material loss since

$$
\begin{aligned}
\mathbf{K}_{0}\left(\bar{\varepsilon}_{r}\right)=\mathbf{K}_{0}^{\prime}\left(\varepsilon_{r}\right), & \mathbf{M}\left(\bar{\varepsilon}_{r}\right)=\mathbf{M}^{\prime}\left(\varepsilon_{r}\right), \\
\mathbf{K}_{1}\left(\bar{\varepsilon}_{r}\right)=\mathbf{K}_{1}^{\prime}\left(\varepsilon_{r}\right), & \mathbf{K}_{2}\left(\bar{\varepsilon}_{r}\right)=\mathbf{K}_{2}^{\prime}\left(\varepsilon_{r}\right) .
\end{aligned}
$$

The eigenvalue problem considering material gain is identical to the adjoint eigenvalue problem stated in Eq. (9) when considering material loss. Hence, the eigen wavenumbers of the guided modes when considering material gain are the conjugates of the corresponding eigen wavenumbers for material loss, as shown in Fig. 3, and the reduction of the group index thus depends only on the magnitude of the imaginary part. This phenomenon can be explained by the amplitude mismatch in multiply scattered light due to material loss/gain, which results in an interference pattern and causes the group index to stay finite [31].
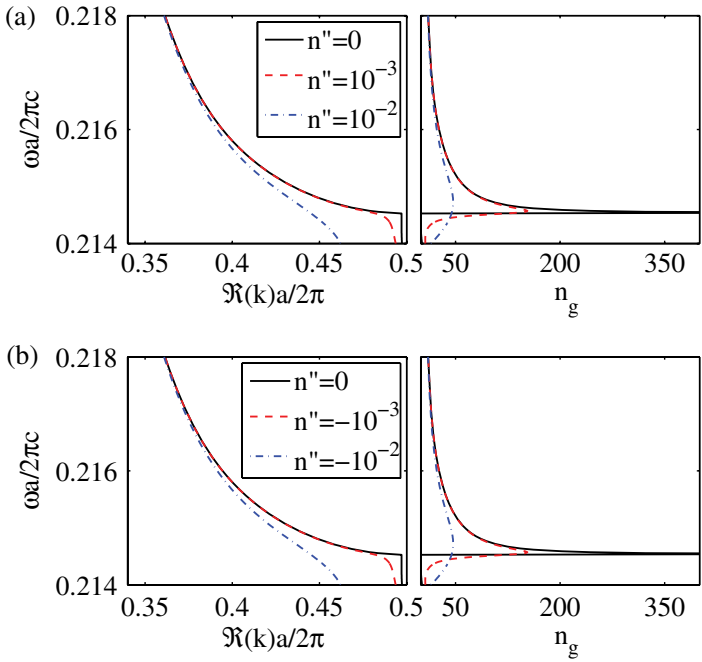

Fig. 2. (Color online) Influence of material loss/gain on the performance of waveguides. (a) Influence of material loss. The solid curve represents the guided band of the lossless waveguide, the dashed curve shows the guided band when considering material loss of $n^{\prime \prime}=10^{-3}$, and the dashed-dotted curve shows the guided band of $n^{\prime \prime}=10^{-2}$. (b) Influence of material gain. The solid curve shows the guided band of the lossless waveguide, the dashed curve represents the guided band of the waveguide with the material gain of $n^{\prime \prime}=-10^{-3}$, and the dashed-dotted curve denotes the guided band when considering the material gain of $n^{\prime \prime}=-10^{-2}$.

\section{Design Parameterization}

A set of design variables $\mathbf{x}$ is now introduced to control and optimize the geometry of the waveguide.

Using both the MMOS approach and the free-topology density approach, we employ $\mathbf{x}$ indirectly to control elementwise physical densities $\bar{\rho}$ that govern the dielectric constant in each element according to

$$
\varepsilon_{e}=\varepsilon_{1}+\bar{\rho}_{e}(\mathbf{x})\left(\varepsilon_{2}-\varepsilon_{1}\right) .
$$

Here, $\varepsilon_{e}$ denotes the permittivity of an element, $e, \varepsilon_{2}=$ $(3.467)^{2}$ denotes the permittivity of $\mathrm{Si}$, and $\varepsilon_{1}=1$ denotes the permittivity of air.

The physical density of element $e$ is found from a threshold projection based on an intermediate element variable $\tilde{\rho}_{e}$ $[\underline{20}, \underline{21}]:$

$$
\bar{\rho}_{e}=\frac{\tanh (\beta \eta)+\tanh \left(\beta\left(\tilde{\rho}_{e}-\eta\right)\right)}{\tanh (\beta \eta)+\tanh (\beta(1-\eta))},
$$

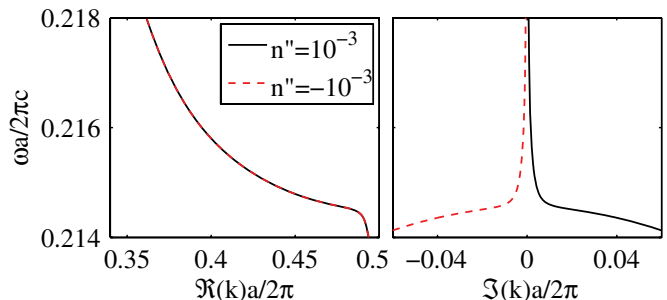

Fig. 3. (Color online) Characteristics of guided bands considering material loss or gain. The solid curves show the characteristics of the guided band in the waveguide with material loss, and the dashed curves show the characteristics of the guided band in the waveguide with the same amount of material gain. 
where $\eta$ is the threshold and $\beta$ is a regularization parameter. The variable $\tilde{\rho}_{e}$ is computed as a filtered value:

$$
\tilde{\rho}_{e}=\frac{\sum_{j \in N e} w\left(\mathbf{r}_{j}\right) v_{j} \rho_{j}}{\sum_{j \in N e} w\left(\mathbf{r}_{j}\right) v_{j}},
$$

where $\mathbf{r}_{j}$ and $v_{j}$ are the centroid and the volume of element $j$, $N_{e}$ is the neighborhood of element $e$ within a certain filter radius $r$ specified by

$$
N_{e}=\left\{j \mid\left\|\mathbf{r}_{j}-\mathbf{r}_{e}\right\| \leq r\right\},
$$

and $w\left(\mathbf{r}_{j}\right)$ is the weight factor of element $j$, calculated by $w\left(\mathbf{r}_{j}\right)=r-\left\|\mathbf{r}_{j}-\mathbf{r}_{e}\right\|$.

With the free-topology density approach, the variable $\rho_{e}$ that appears in Eq. (14) is used directly as the element design variable $x_{e}$. However, in MMOS, the elementwise quantities are projected using a set of circles, where $\rho_{e}=0$ when element $e$ is within circles and $\rho_{e}=1$ when $e$ is outside of circles. Here, radii and locations of the circles are used as design variables. The detailed mathematical description of MMOS can be found in [18].

Backscattering induced by manufacturing imperfections has been reported to be the dominant loss mechanism in the slow-light regime and has to be taken into account in the design procedure. In order to mimic the loss induced by surface roughness, we introduce an edge-related dissipation based on a normalized elementwise quantity $\nu_{e}$ that represents the interface between dielectric material and air. Based on Eq. (13), we compute $\nu_{e}$ as the normalized derivative of the physical density with respect to the filter density, given by $\nu_{e}=1-\left(\tanh \left(\beta\left(\tilde{\rho}_{e}-\eta\right)\right)\right)^{2}$, and the interface lies where $\nu_{e}>0$.

The final elementwise permittivity is now interpolated by modifying Eq. (12) as

$$
\varepsilon_{e}=\varepsilon_{1}+\bar{\rho}_{e}\left(\varepsilon_{2}-\varepsilon_{1}\right)+i \nu_{e}^{p} \varepsilon_{0}^{\prime \prime}
$$

with $p=0.5$ and $\varepsilon_{0}^{\prime \prime}=0.1$. It must be pointed out here that the value $\varepsilon^{\prime \prime}=0.1$ is chosen arbitrarily to quantify the propagation attenuation in different PhCWs for comparisons and the magnitude of $\varepsilon_{0}^{\prime \prime}$ does not affect the optimized designs and the conclusions in the paper.

\section{Optimization Formulations}

In addition to loss engineering of the waveguides, we study waveguides that are optimized with respect to dispersion to realize a prescribed dispersion function. Here we focus on dispersion-engineered slow-light waveguides with a prescribed group index. The optimization problem for the dispersion engineering is described in detail in [20] and can be formulated as

$$
\min _{\mathbf{x}} \max _{\omega_{i}} f\left(\bar{\rho}, \omega_{i}\right)=\left(n_{g}\left(\bar{\rho}, \omega_{i}\right) / n_{g}^{*}-1\right)^{2}
$$

Here, $\omega_{i} \in\left[\omega_{1}, \omega_{2}\right]$ is the target frequency with $\left[\omega_{1}, \omega_{2}\right]$ being the design frequency range, $n_{g}\left(\bar{\rho}, \omega_{i}\right)$ is the actual group index, $n_{g}^{*}$ is the prescribed group index, and $\mathbf{x}$ denotes the set of the design variables considered.

Previous studies have illustrated that the propagation losses of slow-light modes can be engineered through changing the field intensity profiles $[\underline{25}, \underline{28}]$. In the $k(\omega)$ formulation, the propagation losses are represented by the imaginary part of the wavenumber; hence, the $k(\omega)$ formulation enables loss engineering of slow-light waveguides by minimizing either the average propagation loss of the designed modes or by minimizing the maximal propagation loss of the designed modes. The first approach emphasizes the average behavior in the designed frequency range, while the latter one has emphasis on the largest propagation loss within the designed frequency range. In the current work, we employ the first variant and formulate the optimization problem as

$$
\begin{array}{rl}
\min _{\mathbf{x}} & f(\bar{\rho})=\sum_{\omega_{i}}\left(\mathfrak{I}\left(k\left(\bar{\rho}, \omega_{i}\right)\right)\right) \\
\text { s.t. } & \left(n_{g}\left(\bar{\rho}, \omega_{i}\right) / n_{g}^{*}-1\right)^{2}<e_{r}^{2} .
\end{array}
$$

Here, $0 \leq e_{r} \leq 1$ is the relative group index error, indicating the allowed GVD.

For both optimization problems, the presence of multiple guided modes at a given frequency may result in intermodal scattering $[\underline{11}, \underline{20}]$. In order to avoid this, an additional band separation constraint is added to the optimization problems. It is problematic to classify bands in the order of increasing frequency with the $k(\omega)$ formulation and, therefore, the band separation constraints are implemented using the $\omega(k)$ formulation:

$$
\mathfrak{R}\left(\omega_{n-1}\left(k_{i i}\right)\right) \leq a_{1} \min \left(\omega_{i}\right), \quad \mathfrak{R}\left(\omega_{n+1}\left(k_{i i}\right)\right) \geq a_{2} \max \left(\omega_{i}\right),
$$

where $k_{i i}$ is the specified wavenumber in the range $[0, \pi / a], n$ is the order of the designed band, and $a_{1}$ and $a_{2}$ are prescribed numbers defining how far the designed band is separated from the other bands. However, this implies that, in the case of loss engineering, both a $k(\omega)$ and an $\omega(k)$ eigenvalue problem need to be solved.

\section{E. Sensitivity Analysis}

In order to apply gradient-based optimizations, we need to compute the sensitivities of the group index $n_{g}\left(\bar{\rho}, \omega_{i}\right)$ and of the loss $\mathfrak{\Im}\left(k\left(\bar{\rho}, \omega_{i}\right)\right)$.

Although we can use adjoint sensitivity analysis to compute the sensitivity of $n_{g}$ directly, this will be quite costly. Instead, we compute the group index by the finite difference method given by

$$
n_{g}=\frac{\Delta k}{\Delta \omega}=\frac{\mathfrak{R}\left(k\left(\omega_{i}\right)\right)-\mathfrak{R}(k(\omega))}{\omega_{i}-\omega},
$$

which is accurate when $\omega$ is sufficiently close to $\omega_{i}$. The corresponding sensitivity of the group index is then obtained using Eq. ():

$$
\frac{\partial n_{g}}{\partial \bar{\rho}_{e}}=\left(\mathfrak{R}\left(\frac{\partial k\left(\omega_{i}\right)}{\partial \bar{\rho}_{e}}\right)-\mathfrak{R}\left(\frac{\partial k(\omega)}{\partial \bar{\rho}_{e}}\right)\right) /\left(\omega_{i}-\omega\right) .
$$

The sensitivity of the propagation losses $\mathfrak{\Im}(k)$ with respect to the physical density $\bar{\rho}_{e}$ is calculated by 


$$
\frac{\partial \Im(k)}{\partial \bar{\rho}_{e}}=\Im\left(\frac{\partial k}{\partial \bar{\rho}_{e}}\right) .
$$

The sensitivity of eigenvalues from the $\omega(k)$ formulation is calculated following the same procedure as in Eq. (8) when the eigenvalue is distinct, and the sensitivity of multiple eigenvalues is derived following [32].

Finally, the sensitivity of objective and constraints with respect to the design variable $x_{e}$ is obtained using the chain rule [33]:

$$
\frac{\partial f}{\partial x_{e}}=\frac{\partial f}{\partial \bar{\rho}_{e}} \frac{\partial \bar{\rho}_{e}}{\partial x_{e}} .
$$

Here, $\partial \bar{\rho}_{e} / \partial x_{e}$ can be easily derived in the two different parameterizations.

Based on sensitivity analysis, the design is iteratively updated using a gradient-based algorithm, the globally convergent version of the method of moving asymptotes (GCMMA) from Svanberg [34]. A detailed account of optimization procedure can be found in [21]. In the optimization procedure, the regularization parameter $\beta$ is fixed and set to $\beta=16$.

The optimization procedure is implemented in MATLAB and runs in parallel using a standard eight-processor PC. The optimization for dispersion engineering converges within 200 GCMMA iterations, and the one for loss engineering converges within 500 iterations. Each GCMMA iteration includes two to three inner iterations and takes 1-2 min for 16 design wavenumbers.

\section{RESULTS}

In this section, we first investigate the loss mechanism by studying the propagation losses in fixed-topology waveguides and then evaluate the feasibility of loss engineering in the waveguides, comparing the cases of fixed- and free-topology using two parameterizations. Furthermore, we design freetopology loss-engineered slow-light waveguides with enlarged GBP for different group indices and compare them with corresponding fixed-topology loss-engineered waveguides.

\section{A. Propagation Losses of Fixed-Topology Waveguides} We first investigate the propagation loss in several fixedtopology dispersion-engineered waveguides designed for $n_{g}^{*}=50$ when subject to the edge-related dissipation discussed in Subsection 2.C. We consider designs obtained using dispersion engineering with an $\omega(k)$ formulation in the absence of dissipation and with a fixed group index $n_{g}^{*}=50$ [18]. The design variables are the radii and the lateral (normal to the waveguide direction) and longitudinal (along the waveguide direction) locations of the first two rows of air holes using MMOS, with an initial design as in Fig. 1. In this paper, we focus on symmetrical waveguides, where the symmetry along the waveguide direction is enforced by setting the same design variables in the symmetrical locations as in the optimization procedure. However, asymmetrical waveguides can also be engineered to reduce GVD of slow light [35,36].

Figures $4(\mathrm{a})-4(\mathrm{c})$ show the designs obtained from radius and lateral- and longitudinal-location tuning. The performance of the waveguides is listed in Fig. 4(d), in terms of group index and propagation loss. For each waveguide, the propagation loss variation versus frequency coincides with the group index (a)

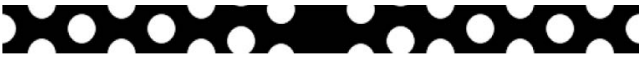

(b) $\mathrm{SO}_{\mathrm{O}} \mathrm{O} \mathrm{O} \mathrm{O}$
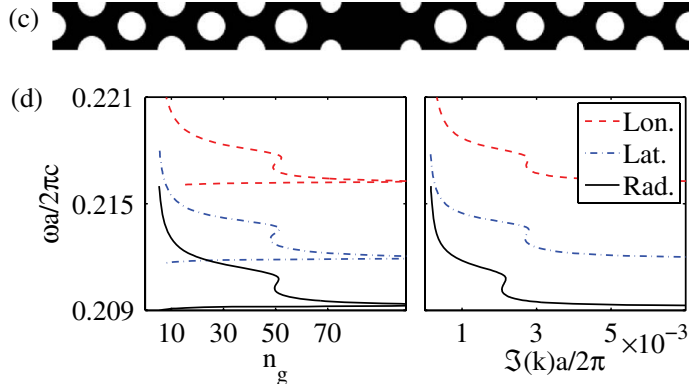

Fig. 4. (Color online) Fixed-topology dispersion-engineered waveguides for $n_{g}^{*}=50$. (a) Longitudinal-location-tuned waveguide. (b) Lateral-location-tuned waveguide. (c) Radius-tuned waveguide. (d) Performance of different waveguides. Left panel: group index versus frequency. Right panel: propagation loss versus frequency. The dashed curves represent the performance of the longitudinallocation-tuned waveguide, the dashed-dotted curves represent the performance of the lateral-location-tuned waveguide, and the solid curvess represent the performance of the radius-tuned waveguide.

variation versus frequency. Thus, a high group index seems to be intrinsically linked to high propagation losses.

We employ the GBP and the average propagation loss $(\langle\mathfrak{I}(k)\rangle)$ as figures of merit to evaluate the performance of different waveguides. The GBP is defined by

$$
\mathrm{GBP}=\Delta \omega\left\langle n_{g}\right\rangle / \omega_{0},
$$

where $\Delta \omega$ is the frequency range where the GVD is negligible, and $\left\langle n_{g}\right\rangle$ and $\omega_{0}$ are the corresponding average group index and the center frequency. In practice, a relative group index error of $\pm 10 \%$ is utilized to indicate the negligible GVD region.

The detailed performances of the waveguides are summarized in Table 1 . Under $\pm 10 \%$ criterion, all the waveguides achieve a GBP of 0.29 or above. The average propagation losses differ for the different waveguides and the radius-tuned waveguide exhibits the lowest average propagation loss among them.

In order to further study the loss mechanism, we employ the concepts of energy density $\mathbf{I}_{s}$ and dissipative energy density $\mathbf{I}_{d}$ [37], given by

$$
\mathbf{I}_{s}=\frac{1}{2} \varepsilon_{0} \varepsilon_{r}^{\prime}|\mathbf{E}|^{2}, \quad \mathbf{I}_{d}=\frac{1}{2} \varepsilon_{0} \varepsilon_{r}^{\prime \prime}|\mathbf{E}|^{2}
$$

Here, $\mathbf{E}$ is the electric field calculated by $\mathbf{E}=1 /\left(i \omega \varepsilon_{0} \varepsilon_{r}\right) \nabla \times \mathbf{H}$ with $\mathbf{H}=\left[\begin{array}{lll}0 & 0 & h\end{array}\right]$, and $\varepsilon_{0}$ is the permittivity in vacuum.

Table 1. Performance Comparison of Fixed-Topology Waveguides ${ }^{a}$

\begin{tabular}{lcccc}
\hline Des. & $\omega_{0} a / 2 \pi c$ & $\left\langle n_{g}\right\rangle$ & GBP & $\langle\Im(k)\rangle a / 2 \pi\left(10^{-3}\right)$ \\
\hline Lon. & 0.2172 & 50.54 & 0.29 & 2.78 \\
Lat. & 0.2131 & 49.77 & 0.31 & 2.75 \\
Rad. & 0.2104 & 50.50 & 0.33 & 2.12 \\
\hline
\end{tabular}

${ }^{a}$ The first row represents the longitudinal (along the waveguide direction)location-tuned waveguide, the second row represents the lateral (normal to the waveguide direction)-location-tuned waveguide, and the third row represents the radius-tuned waveguide. 
(a)
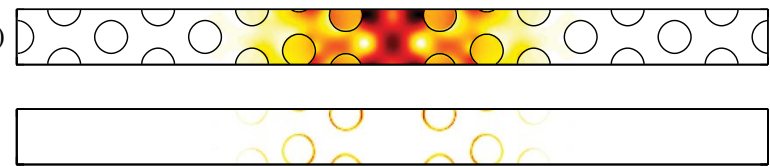

(b)
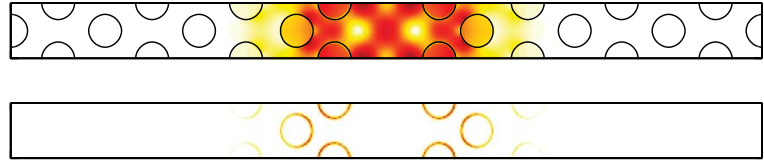

(c)
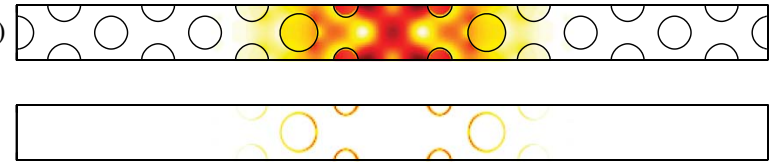

Fig. 5. (Color online) Energy density and dissipative energy density of slow-light modes with $n_{g} \approx 50$ in fixed-topology dispersionengineered waveguides. (a) Longitudinal-location-tuned waveguide. Top: energy density. Bottom: dissipative energy density. (b) Laterallocation-tuned waveguide. (c) Radius-tuned waveguide.

Figure 5 shows the energy density, $\mathbf{I}_{s}$, and dissipative energy density, $\mathbf{I}_{d}$, of slow-light modes with $n_{g} \approx 50$ in different waveguides. Compared to the radius-tuned waveguide, the longitudinal-location-tuned waveguide has a smaller waveguide core, and hence exhibits a stronger field intensity at the waveguide core, which results in larger propagation losses on the interface of the first row of air holes. Despite a similar size of waveguide core as the radius-tuned waveguide, stronger field intensity originating from the second row of air holes can be observed in the lateral-location-tuned waveguide, hence causing more propagation losses on the second row of air holes. Furthermore, the radius-tuned waveguide has the shortest interface among all the designs, which also results in a smaller fraction of field available for scattering at imperfections. The detailed studies on the energy density and dissipative energy density illustrate that the smallest propagation loss is found with the smallest fraction of field intensity involved in scattering at imperfections. This is in agreement with recent theoretical and experimental studies [25,28] and supports our phenomenological representation of disorderinduced scattering losses by the edge-related dissipation.

\section{B. Loss-Engineered Slow-Light Waveguides}

From the conducted studies, it is apparent that it is possible to manipulate the loss while maintaining high values for $n_{g}$ and GBP, and, in this section, we study the feasibility of loss engineering slow-light waveguides by minimizing the average propagation losses of the designed slow-light modes using the optimization formulation presented in Eq. (18). The initial waveguide is the radius-tuned waveguide with $n_{g}^{*}=50$. The design frequency range is set to be $\omega_{i} \in[0.2097,0.2112] 2 \pi c / a$ with 16 design frequencies. In order to ensure that the average group index of the optimized waveguide is equal to or larger than $n_{g}^{*}$ in the design frequency range, a constraint on the average group index is implemented as $\left\langle n_{g}\right\rangle \geq n_{g}^{*}$.

The free-topology loss-engineered waveguides are obtained using the density approach with the design domain being the first three and a half rows of air holes adjacent to the waveguide core, shown in Fig. 6. The fixed-topology lossengineered waveguides are obtained using MMOS with the

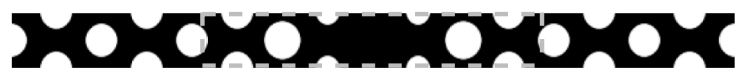

Fig. 6. Initial design and the design domain. The gray dashed rectangle indicates the design domain with a symmetry along the waveguide direction.

design variables as locations and radii of the first two rows of air holes.

The free-topology loss-engineered waveguides for relative group index errors of $e_{r}=10 \%$ and $e_{r}=5 \%$ are shown in Figs. $7(\mathrm{a})$ and $7(\mathrm{~b})$, respectively. Figure $\underline{7(\mathrm{c})}$ depicts the fixedtopology loss-engineered waveguide for $e_{r}=5 \%$. The performances of the different waveguides are depicted in Fig. $7(\mathrm{~d})$. As expected, the group index variation in the free-topology loss-engineered waveguide of $e_{r}=10 \%$ is much larger than that in the other waveguides, including the initial design. Under the same relative group index error of $e_{r}=5 \%$, the freeand fixed-topology loss-engineered waveguides deviate slightly from each other and achieve equivalent performance in terms of group index variation and propagation losses. Compared to the initial design shown in Fig. 4(c), a noticeable improvement of the propagation losses can be observed in both waveguides.

The detailed performances of different waveguides are summarized in Table 2 . Compared with the initial waveguide, the loss-engineered designs achieve observable lower average propagation losses. For the same group index, the freetopology loss-engineered waveguide achieves a slightly smaller propagation loss than the fixed-topology loss-engineered waveguide and the average propagation loss can be further reduced by allowing larger GVD. The energy density and dissipative energy density of slow-light modes of $n_{g} \approx 50$ in different waveguides (shown in Fig. $\underline{\text { ) }}$ illustrate that the reduction of the propagation loss is attributed to the smaller field intensity at the interface for the free-topology lossengineered waveguide with $e_{r}=10 \%$ and the reduction of

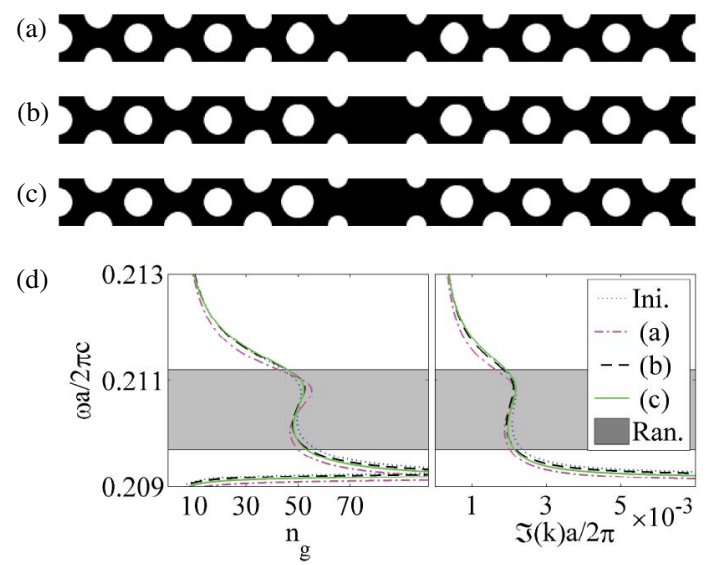

Fig. 7. (Color online) Loss-engineered slow-light waveguides for $n_{g}^{*}=50$. (a) Free-topology loss-engineered waveguide for $e_{r}=$ 10\%. (b) Free-topology loss-engineered waveguide with $e_{r}=5 \%$. (c) Fixed-topology loss-engineered waveguide for $e_{r}=5 \%$. (d) Performances of different waveguides. The dotted curves show the performance of the initial waveguide, the dashed-dotted curves show the performance of the free-topology loss-engineered waveguide for $e_{r}=10 \%$, the dashed curves show the performance of the freetopology loss-engineered waveguide $e_{r}=5 \%$, and the solid curves show the performances of the fixed-topology loss-engineered waveguides. The gray regions indicate the design range. 
Table 2. Performance Comparison of LossEngineered Waveguides with $n_{g}^{*}=50^{a}$

\begin{tabular}{lcccc}
\hline Des. & $\omega_{0} a / 2 \pi c$ & $\left\langle n_{g}\right\rangle$ & GBP & $\langle\mathfrak{\Im}(k)\rangle a / 2 \pi\left(10^{-3}\right)$ \\
\hline Fre. Top. 10\% & 0.2102 & 49.95 & 0.37 & 1.98 \\
Fre. Top. 5\% & 0.2103 & 50.06 & 0.35 & 2.01 \\
Fix. Top. 5\% & 0.2103 & 50.16 & 0.36 & 2.05 \\
\hline
\end{tabular}

${ }^{a}$ The first row shows the performance of the free-topology loss-engineered waveguide of $e_{r}=10 \%$, the second row shows the performance of the freetopology loss-engineered waveguide of $e_{r}=5 \%$, and the third row shows the performance of the fixed-topology loss-engineered waveguide of $e_{r}=5 \%$.

the propagation loss in the loss-engineered waveguides for $e_{r}=5 \%$ is mainly attributed to a smaller interface between air and $\mathrm{Si}$ in the first row. However, due to the fact that the modal profiles are dominated by the group index, i.e., the higher the group index, the larger the modal profile, the improvement of the propagation losses is restricted.

\section{Loss-Engineered Slow-Light Waveguides with Enlarged GBP}

The capacity of slow-light waveguides, i.e., GBP, is crucial for the applications. Previous studies have illustrated that free-topology dispersion-engineered waveguides can achieve significantly larger GBP compared with fixed-topology dispersion-engineered waveguides [18]. In this section, we design free-topology loss-engineered waveguides with an enlarged GBP. The optimization procedure is accomplished in two steps. Dispersion engineering is first performed to enhance the GBP using the optimization formulation presented in Eq. (17). Using the dispersion-engineered slow-light waveguide as an initial design, we further perform loss engineering to reduce the average propagation loss of the designed slowlight modes under a relative group index error of $e_{r}=5 \%$.

As in the first example, we aim at designing a slowlight waveguide for $n_{g}^{*}=50$ in the frequency range of $\omega_{i} \in[0.2103,0.2123] 2 \pi c / a$. The frequency range is chosen to ensure a GBP of 0.45 for the optimized waveguides. The initial design is chosen as the radius-tuned waveguide shown in Fig. 6. The dispersion-engineered waveguide and lossengineered waveguide are shown in Figs. 9(a) and 9(b), respectively. Figure 9 (c) depicts the performance of the

(a)
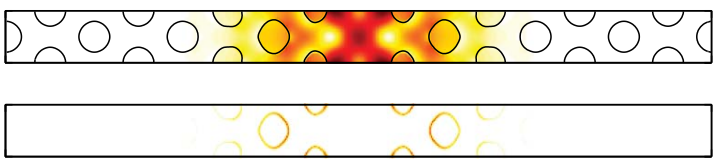

(b)
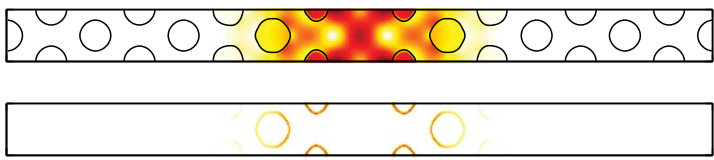

(c)
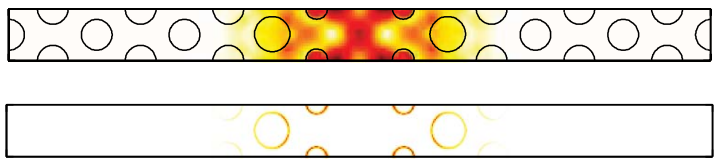

Fig. 8. (Color online) Energy density and dissipative energy density of slow-light modes with $n_{g} \approx 50$ in the optimized waveguides. (a) Free-topology loss-engineered waveguide for $e_{r}=10 \%$. (b) Freetopology loss-engineered waveguide for $e_{r}=5 \%$. (c) Fixed-topology loss-engineered waveguide for $e_{r}=5 \%$. (a)

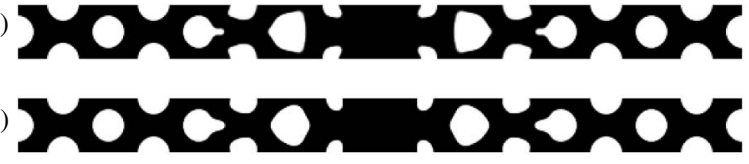

(c)

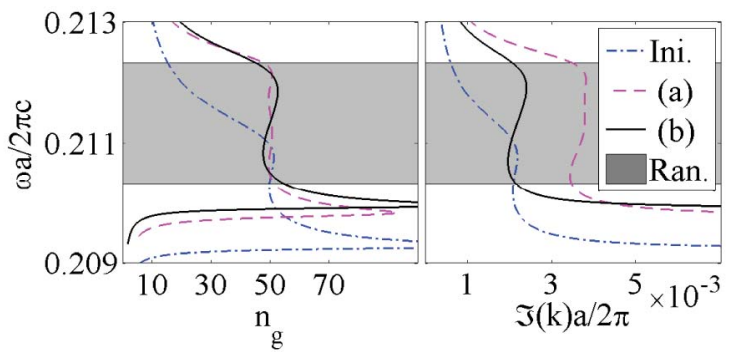

Fig. 9. (Color online) Free-topology optimized slow-light waveguides with an enlarged GBP for $n_{g}^{*}=50$. (a) Free-topology dispersion-engineered waveguide. (b) Free-topology loss-engineered waveguide. (c) Group index and loss of different waveguides. The dashed-dotted curves represent the performance of the initial design, the dashed curves represent the performances of the dispersionengineered waveguide, and the solid curves represent the performance of the loss-engineered waveguides. The gray regions indicate the design range.

optimized waveguides. Compared with the radius-tuned waveguide, the free-topology optimized waveguides achieve a much larger GBP. However, the dispersion-engineered waveguide encounters significantly higher propagation losses due to the sharp features in the waveguide shown in Fig. 9(a) and the bigger modal size shown in Fig. 10(a). Thus, a significant dissipation energy density can be observed on the third rows of air holes in the dispersion-engineered design. In contrast to the dispersion-engineered slow-light waveguide, the lossengineered slow-light waveguide has a smooth interface between air and dielectric material and the corresponding modal size is relatively small. Hence, the propagation losses have been significantly reduced in the loss-engineered waveguide and are equivalent to the ones in the radius-tuned slow-light waveguide [see Fig. 9(c)].

The characteristics of the dispersion-engineered waveguide and loss-engineered waveguide are summarized in Table 3. The GPB of the waveguides has been improved by a factor of 1.42 or above compared to the initial design. Furthermore, through loss engineering, the free-topology engineered slowlight waveguide can achieve an equally good performance in terms of propagation losses with the radius-tuned waveguide. Compared with the loss-engineered waveguides presented in the previous subsection, the GBP of the slow-light waveguides

(a)
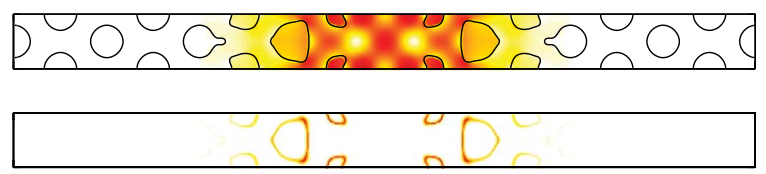

(b)
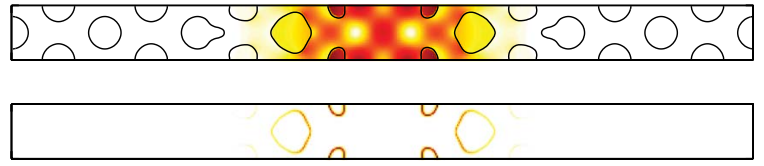

Fig. 10. (Color online) Energy density and dissipative energy density of slow-light modes with $n_{g} \approx 50$ in different waveguides. (a) Dispersion-engineered waveguide of $n_{g}^{*}=50$. (b) Loss-engineered waveguide of $n_{g}^{*}=50$. 
Table 3. Performance Comparison of the Optimized Waveguides with $n_{g}^{*}=50$ and Enlarged GBP ${ }^{a}$

\begin{tabular}{lcccc}
\hline Des. & $\omega_{0} a / 2 \pi c$ & $\left\langle n_{g}\right\rangle$ & GBP & $\langle\mathfrak{\Im}(k)\rangle a / 2 \pi\left(10^{-3}\right)$ \\
\hline Dis. & 0.2111 & 50.27 & 0.50 & 3.62 \\
Los. & 0.2112 & 49.82 & 0.47 & 2.13 \\
\hline
\end{tabular}

${ }^{a}$ The first row shows the performance of the dispersion-engineered waveguide, and the second row depicts the performance of the lossengineered waveguide.

has been improved by a factor of 1.27 with an acceptable sacrifice of the propagation losses.

In order to further compare the fixed-topology lossengineered waveguides and free-topology loss-engineered waveguides, we design loss-engineered waveguides for different group indices in the interval of $n_{g}^{*} \in[40,100]$. The same initial design, i.e., that of Fig. 6 , is chosen for all the target group indices. The target frequency ranges of free-topology loss-engineered waveguides are chosen to ensure a GBP of 0.45 with a starting frequency of $\omega_{1}=0.21032 \pi c / a$, and the target frequency ranges of fixed-topology loss-engineered waveguides are chosen to ensure a GBP of 0.33 with the same starting frequency.

Figure 11 shows the characteristics of the fixed-topology loss-engineered waveguides and the free-topology lossengineered waveguides in terms of average propagation losses and GBP. All the fixed-topology loss-engineered waveguides achieve a GBP of 0.33 or above, and all the free-topology loss-engineered waveguides can achieve a GBP of 0.45 or above. For the same target group index, the free-topology loss-engineered waveguide displays higher average propagation loss than the fixed-topology loss-engineered waveguides. Hence, the enhancement of GBP is at the cost of the average propagation loss. It is noted that the average propagation loss increases with the target group index in both cases, which indicates that the light slowdown in itself is a dominant factor in determining the resulting losses.

Figure 12 depicts the characteristics of the fixed- and free-topology loss-engineered waveguides for $n_{g}^{*}=90$. It is seen that, at low frequencies, the slow-light modes in the free-topology loss-engineered waveguide exhibit equivalent propagation losses as the ones in the fixed-topology lossengineered waveguide, and at high frequencies, the slow-light modes exhibit much higher propagation losses than

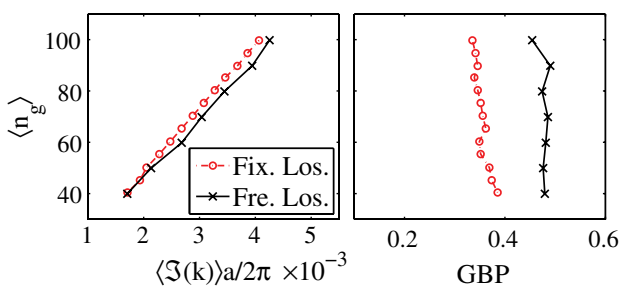

Fig. 11. (Color online) Comparison between fixed- and free-topology loss-engineered waveguides for different group indices. Left panel: average group index versus average propagation loss. Right panel: average group index versus GBP. The dashed-dotted curves represent the performance of the fixed-topology loss-engineered waveguides with circles indicating the different designs considered. The solid curves represent the performance of the loss-engineered slow-light waveguides, with crosses indicating the different designs considered. (a)

(b)

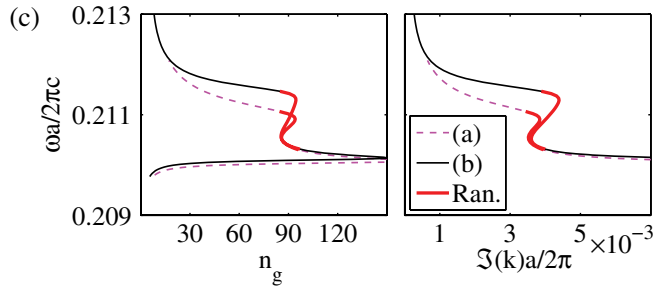

Fig. 12. (Color online) Loss-engineered waveguides for $n_{g}^{*}=90$ (a) Fixed-topology loss-engineered waveguide. (b) Free-topology loss-engineered waveguide. (c) Performance of different waveguides. The dashed curves represent the performance of the fixed-topology loss-engineered waveguide, the black solid curves represent the freetopology loss-engineered waveguides, and the bold red center parts of the solid curves represent the design ranges.

encountered in the fixed-topology loss-engineered waveguides. Hence, the large average propagation loss in the free-topology loss-engineered waveguide is attributed to the large propagation losses of slow-light modes with high frequencies. The energy densities of two design slow-light modes with $n_{g} \approx 94$ in the free-topology loss-engineered waveguide (seen in Fig. 13) reveal that the slow-light mode with high frequency has much larger modal size compared to the other slow-light mode. This leads to a significant increase in the propagation loss.

In order to further evaluate the performance of the lossengineered waveguides, we calculate the transmission $(T)$ of the engineered waveguides of $n_{g}^{*}=90$ in the presence of the edge-related loss, consisting of 20 periods of supercells. The transmission is found from the time-averaged Poynting vector, i.e., the component in the waveguide direction [38]. The transmission is normalized with respect to the transmission of the corresponding ideal waveguides. The normalized transmission spectra of the free-topology dispersionengineered waveguide and the fixed- and free-topology lossengineered waveguides are shown in Fig. 14. As illustrated by the complex band structure, the transmission has been significantly enhanced through the loss engineering. A significant drop can be observed at high frequency in the transmission spectrum of the free-topology loss-engineered waveguide. This observation supports the propagation losses evaluated from the $k(\omega)$ formulation.

(a)
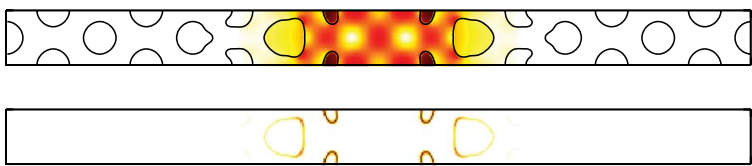

(b)
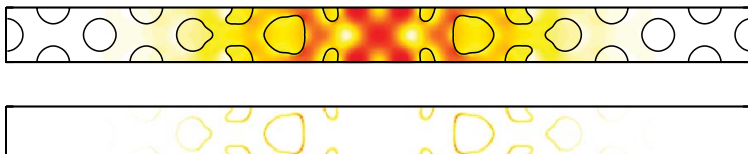

Fig. 13. (Color online) Energy density and dissipative energy density of slow-light modes with $n_{g} \approx 94$ at different frequencies in the freetopology loss-engineered waveguide for $n_{g}^{*}=90$. (a) $\omega a / 2 \pi=0.21034$ (b) $\omega a / 2 \pi=0.21123$. 


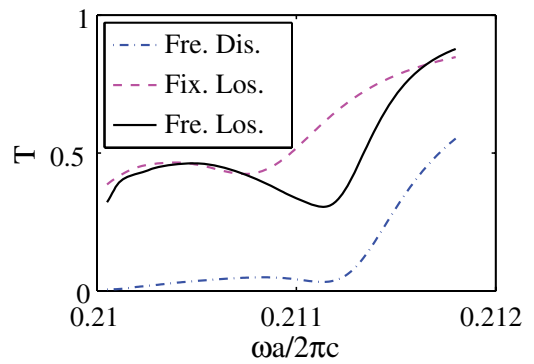

Fig. 14. (Color online) Transmission spectra of the loss-engineered waveguides of 20 periods in the presence of the edge-related loss. The dashed-dotted curve represents the transmission spectrum of the free-topology dispersion-engineered waveguide, the dashed curve represents the one of the fixed-topology loss-engineered waveguide, and the solid curve represents the one of the free-topology loss-engineered waveguide.

\section{CONCLUSIONS}

In this study, systematic loss engineering has been performed to reduce the propagation losses of slow-light waveguides. In order to mimic scattering losses induced by manufacturing imperfections, we have utilized the concept of the effective dissipation, where the scattering losses have been represented by an edge-related dissipation. The propagation losses of slow-light modes have been evaluated by the imaginary part of eigenvalues in the complex band structure calculation, which was supported by transmission computations for a finite waveguide.

Numerical studies on the propagation losses of circularhole-based waveguides obtained by radius and location tuning have shown that loss engineering can be realized by reducing the fraction of field available for scattering. Two parameterizations have been utilized to describe the supercell, i.e., the material mask overlay strategy for the fixed-topology waveguides and the density approach for the free-topology waveguides. By minimizing the average propagation losses, the loss-engineered waveguides from these two parameterizations have achieved smaller propagation losses while maintaining equivalent performance in terms of GVD and GBP as the initial design. However, the improvement of the propagation losses is inherently restricted due to the limited freedom on the manipulation of the modal profiles for a given group index.

Loss-engineered slow-light waveguides with enlarged GPB have been designed in two steps. First, the dispersion engineering has been performed to enhance the GBP and then the loss engineering has been employed to reduce the propagation losses of the dispersion-engineered waveguides caused by the sharp features in the design or/and the large modal size. Numerical examples for $n_{g}^{*}=50$ have illustrated that the propagation losses can be significantly reduced in the freetopology engineered waveguides by the loss engineering. Detailed comparisons between fixed- and free-topology loss-engineered waveguides for different target group indices have illustrated that the enhancement of GBP in the freetopology loss-engineered waveguides have been realized at the expense of propagation losses. Further studies on the freetopology loss-engineered waveguide have revealed that the larger propagation losses in the free-topology loss-engineered waveguides are due to the large propagation losses of slowlight modes with high frequencies. The proper trade-off between propagation losses and GBP will depend on the specific application.

The presented approach can be extended in a straightforward way to the loss engineering of $3 \mathrm{D}$ waveguides, where $2 \mathrm{D}$ loss-engineered waveguides will be good starting points. The out-of-plane radiation can be taken into account in the $3 \mathrm{D}$ loss-engineered waveguides by using a proper boundary condition in the additional direction. However, increased dimensionality will increase the computing time significantly and introduce challenges regarding efficient large scale eigenvalue solvers.

\section{ACKNOWLEDGMENTS}

This work was financially supported by Villum Fonden (via the NATEC Centre of Excellence) and by the Danish Center for Scientific Computing (DCSC). The authors thank Vincent Laude for helpful discussions on the formulation of the $k(\omega)$ eigenvalue problem and fruitful discussions with members of the TopOpt-group (www.topopt.dtu.dk) and the Department of Photonics Engineering at DTU are gratefully acknowledged.

\section{REFERENCES}

1. T. F. Krauss, "Why do we need slow light?" Nat. Photonics 2 448-450 (2008).

2. T. Baba, "Slow light in photonic crystals," Nat. Photonics 2, 465-473 (2008)

3. D. M. Beggs, T. P. White, L. O'Faolain, and T. F. Krauss, "Ultracompact and low-power optical switch based on silicon photonic crystals," Opt. Lett. 33, 147-149 (2008).

4. J. E. McMillan, X. D. Yang, N. C. Panoiu, R. M. Osgood, and C. W. Wong, "Enhanced stimulated Raman scattering in slow-light photonic crystal waveguides," Opt. Lett. 31, 1235-1237 (2006).

5. B. Corcoran, C. Monat, C. Grillet, D. J. Moss, B. J. Eggleton, T. P. White, L. O'Faolain, and T. F. Krauss, "Green light emission in silicon through slow-light enhanced third-harmonic generation in photonic-crystal waveguides," Nat. Photonics 3, 206-210 (2009).

6. M. Heuck, S. Blaaberg, and J. Mork, "Theory of passively modelocked photonic crystal semiconductor lasers," Opt. Express 18, 18003-18014 (2010).

7. J. G. Pedersen, S. S. Xiao, and N. A. Mortensen, "Limits of slow light in photonic crystals," Phys. Rev. B 78, 153101 (2008).

8. J. Grgić, J. R. Ott, F. Wang, O. Sigmund, A.-P. Jauho, J. Mørk, and N. A. Mortensen, "Fundamental limitations to gain enhancement in periodic media and waveguides," Phys. Rev. Lett. 108, 183903 (2012).

9. R. J. P. Engelen, Y. Sugimoto, Y. Watanabe, J. P. Korterik, N. Ikeda, N. F. van Hulst, K. Asakawa, and L. Kuipers, "The effect of higher-order dispersion on slow light propagation in photonic crystal waveguides," Opt. Express 14, 1658-1672 (2006).

10. S. Hughes, L. Ramunno, J. F. Young, and J. E. Sipe, "Extrinsic optical scattering loss in photonic crystal waveguides: role of fabrication disorder and photon group velocity," Phys. Rev. Lett. 94, 033903 (2005).

11. E. Kuramochi, M. Notomi, S. Hughes, A. Shinya, T. Watanabe, and L. Ramunno, "Disorder-induced scattering loss of linedefect waveguides in photonic crystal slabs," Phys. Rev. B 72, 161318 (2005).

12. D. Mori and T. Baba, "Dispersion-controlled optical group delay device by chirped photonic crystal waveguides," Appl. Phys. Lett. 85, 1101-1103 (2004).

13. D. Mori, S. Kubo, H. Sasaki, and T. Baba, "Experimental demonstration of wideband dispersion-compensated slow light by a chirped photonic crystal directional coupler," Opt. Express 15, 5264-5270 (2007).

14. L. H. Frandsen, A. V. Lavrinenko, J. Fage-Pedersen, and P. I. Borel, "Photonic crystal waveguides with semi-slow light and 
tailored dispersion properties," Opt. Express 14, 9444-9450 (2006).

15. J. Li, T. P. White, L. O'Faolain, A. Gomez-Iglesias, and T. F. Krauss, "Systematic design of flat band slow light in photonic crystal waveguides," Opt. Express 16, 6227-6232 (2008).

16. S. A. Schulz, L. O'Faolain, D. M. Beggs, T. P. White, A. Melloni, and T. F. Krauss, "Dispersion engineered slow light in photonic crystals: a comparison," J. Opt. 12, 104004 (2010).

17. T. Kawasaki, D. Mori, and T. Baba, "Experimental observation of slow light in photonic crystal coupled waveguides," Opt. Express 15, 10274-10281 (2007).

18. F. Wang, J. S. Jensen, and O. Sigmund, "High-performance slow light photonic crystal waveguides with topology optimized or circular-hole based material layouts," Photon. Nanostruct. Fundam. Applic. 10, 378-388 (2012).

19. R. Stainko and O. Sigmund, "Tailoring dispersion properties of photonic crystal waveguides by topology optimization," Waves Random Complex Media 17, 477-489 (2007).

20. F. Wang, J. S. Jensen, and O. Sigmund, "Robust topology optimization of photonic crystal waveguides with tailored dispersion properties," J. Opt. Soc. Am. B 28, 387-397 (2011).

21. F. Wang, B. S. Lazarov, and O. Sigmund, "On projection methods, convergence and robust formulations in topology optimization," Struct. Multidisc. Optim. 43, 767-784 (2011).

22. H. Benisty, D. Labilloy, C. Weisbuch, C. J. M. Smith, T. F. Krauss, D. Cassagne, A. Beraud, and C. Jouanin, "Radiation losses of waveguide-based two-dimensional photonic crystals: positive role of the substrate," Appl. Phys. Lett. 76, 532-534 (2000).

23. R. Ferrini, R. Houdre, H. Benisty, M. Qiu, and J. Moosburger, "Radiation losses in planar photonic crystals: two-dimensional representation of hole depth and shape by an imaginary dielectric constant," J. Opt. Soc. Am. B 20, 469-478 (2003).

24. M. Patterson, S. Hughes, S. Schulz, D. M. Beggs, T. P. White, L. O'Faolain, and T. F. Krauss, "Disorder-induced incoherent scattering losses in photonic crystal waveguides: Bloch mode reshaping, multiple scattering, and breakdown of the BeerLambert law," Phys. Rev. B 80, 195305 (2009).

25. L. O'Faolain, S. A. Schulz, D. M. Beggs, T. P. White, M. Spasenovic, L. Kuipers, F. Morichetti, A. Melloni, S. Mazoyer,
J. P. Hugonin, P. Lalanne, and T. F. Krauss, "Loss engineered slow light waveguides," Opt. Express 18, 27627-27638 (2010).

26. L. C. Andreani and D. Gerace, "Light-matter interaction in photonic crystal slabs," Phys. Status Solidi B 244, 3528-3539 (2007).

27. M. Patterson and S. Hughes, "Theory of disorder-induced coherent scattering and light localization in slow-light photonic crystal waveguides," J. Opt. 12, 104013 (2010).

28. A. Petrov, M. Krause, and M. Eich, "Backscattering and disorder limits in slow light photonic crystal waveguides," Opt. Express 17, 8676-8684 (2009).

29. R. Ferrini, D. Leuenberger, R. Houdre, H. Benisty, M. Kamp, and A. Forchel, "Disorder-induced losses in planar photonic crystals," Opt. Lett. 31, 1426-1428 (2006).

30. K. C. Huang, E. Lidorikis, X. Y. Jiang, J. D. Joannopoulos, K. A. Nelson, P. Bienstman, and S. H. Fan, "Nature of lossy Bloch states in polaritonic photonic crystals," Phys. Rev. B 69, 195111 (2004).

31. T. F. Krauss, "Slow light in photonic crystal waveguides," J. Phys. D 40, 2666-2670 (2007).

32. A. P. Seyranian, E. Lund, and N. Olhoff, "Multiple eigenvalues in structural optimization problems," Struct. Optim. 8, 207-227 (1994).

33. O. Sigmund, "Morphology-based black and white filters for topology optimization," Struct. Multidisc. Optim. 33, 401-424 (2007).

34. K. Svanberg, "A class of globally convergent optimization methods based on conservative convex separable approximations," SIAM J. Optim. 12, 555-573 (2002).

35. J. Ma and C. Jiang, "Demonstration of ultraslow modes in asymmetric line-defect photonic crystal waveguides," IEEE Photon. Technol. Lett. 20, 1237-1239 (2008).

36. P. Colman, S. Combrié, G. Lehoucq, and A. De Rossi, "Control of dispersion in photonic crystal waveguides using group symmetry theory," Opt. Express 20, 13108-13114 (2012).

37. F. D. Nunes, T. C. Vasconcelos, M. Bezerra, and J. Weiner, "Electromagnetic energy density in dispersive and dissipative media," J. Opt. Soc. Am. B 28, 1544-1552 (2011).

38. J. S. Jensen and O. Sigmund, "Topology optimization of photonic crystal structures: a high-bandwidth low-loss T-junction waveguide,” J. Opt. Soc. Am. B 22, 1191-1198 (2005). 